\title{
Anticoagulation therapy and invasive management of acute non-ST elevation coronary syndromes: guidelines and everyday practice
}

\author{
Irzal Hadžibegović*, Marijana Knežević Praveček, Krešimir Gabaldo, Božo Vujeva, Đeiti Prvulović
}

*General Hospital "Dr. Josip Benčević", Slavonski Brod, Croatia

Introduction and goal: The latest 2011 non-ST-segment elevation acute coronary syndromes (NSTE-ACS) management guidelines indicated that all patients should receive anticoagulation drugs irrespective of the treatment strategy. In this report, 2-year data on anticoagulation therapies for NSTE-ACS are presented, in relation to invasive management selection and treatment outcomes in a clinical setting without registered bivalirudin.

Patients and Methods: Data for 374 patients with NSTEACS treated in the Coronary Care Unit in General Hospital Slavonski Brod, Croatia were analyzed from January 2012 to December 2013. Treatment strategies, outcomes and complications among patients treated with different anticoagulation agents (enoxaparine, fondaparinux or unfractionated heparin) were analyzed and compared.

Results: In all, $72 \%$ of all NSTE-ACS patients were treated invasively, out of which $66 \%$ underwent angiography within 72 hours. Urgent and early angiography was performed in $44 \%$ of all invasively treated patients. Generally, $58 \%$ and $5 \%$ of patients received enoxaparine and unfractionated heparin (UFH), respectively, whereas fondaparinux was administered in $37 \%$ of patients. Median age of patients treated

Received: $17^{\text {th }}$ Apr 2014

*Address for correspondence: Opća bolnica „Dr. Josip Benčević, Štampareva 42, HR-35000 Slavonski Brod, Croatia.

Phone: +385-35-201-689

E-mail: irzalh@gmail.com with enoxaparine was 66 , compared to 76 in the fondaparinux group. There were significantly more patients treated with enoxaparine and UFH than with fondaparinux in the invasive strategy group. We observed that the number of patients treated with fondaparinux in the invasive group increased together with the period between symptom onset and angiography. The number of patients treated conservatively with either fondaparinux or enoxaparine was almost equal. There were no patients treated conservatively with UFH. In general, in-hospital mortality was low, $2.6 \%$ with no significant differences in bleeding events regarding the selection of anticoagulation therapy.

Conclusions: Although the guidelines gave preference to fondaparinux, everyday practice in our hospital showed that enoxaparine was a preferred agent, particularly in younger patients selected for early invasive strategy, most probably because of the risk/benefit ratio and opportunity of avoiding the mixing of anticoagulation agents. Only $5 \%$ of patients were selected for urgent invasive approach with UFH, which should probably increase in the future. Considering a higher cost of bivalirudin, the introduction of this agent for non-ST segment elevation myocardial infarction management does not seem to be mandatory in our clinical setting, since this small group of patients was adequately managed with UFH.

KEYWORDS: acute coronary syndrome, anticoagulation therapy, percutaneous coronary intervention.

CITATION: Cardiol Croat. 2014;9(5-6):170.

\section{Literature}

1. Hamm CW, Bassand JP, Agewall S, et al; ESC Committee for Practice Guidelines. ESC Guidelines for the management of acute coronary syndromes in patients presenting without persistent ST-segment elevation: The Task Force for the management of acute coronary syndromes (ACS) in patients presenting without persistent ST-segment elevation of the European Society of Cardiology (ESC). Eur Heart J. 2011;32(23):2999-3054.

2. Katritsis DG1, Siontis GC, Kastrati A, et al. Optimal timing of coronary angiography and potential intervention in non-ST-elevation acute coronary syndromes. Eur Heart J. 2011;32:32-40. 